\title{
A Reexamination of the Northern Hemisphere Sea Level Pressure Variability by the Independent Component Analysis
}

\author{
Atsushi Mori ${ }^{1}$, Nobuaki Kawasaki², Kensuke Yamazaki ${ }^{3}$, \\ Meiji Honda ${ }^{4}$, and Hisashi Nakamura ${ }^{4,5}$ \\ ${ }^{1}$ Department of Astronomy and Earth Sciences, Tokyo Gakugei University, Tokyo, Japan \\ ${ }^{2}$ Senior High School at Otsuka of Tsukuba University, Tokyo, Japan \\ ${ }^{3}$ Department of Technology and Information Science, Tokyo Gakugei University, Tokyo, Japan \\ ${ }^{4}$ Frontier Research Center for Global Change, Japan Agency for Marine-Earth Science \\ and Technology Center, Yokohama, Japan \\ ${ }^{5}$ Department of Earth and Planetary Science, the University of Tokyo, Tokyo, Japan
}

\begin{abstract}
The results of the empirical orthogonal function (EOF) analysis applied to the sea-level pressure (SLP) variability in the extratropical Northern Hemisphere is reexamined from viewpoint of statistical independence. For this purpose, "Independent Component Analysis" is introduced, whose effectiveness is demonstrated in its application to a set of simple hypothetical models. While its application to stratospheric variability confirms the relevance of the EOF analysis, its application to the SLP suggests that the two leading components of the EOF analysis are likely superpositions of two statistically independent signals. One of them represents a seesaw-like relationship in the intensities between the surface Aleutian and Icelandic lows, and the other is characterized by a significant long-term trend with the cold ocean-warm land pattern.
\end{abstract}

\section{Introduction}

An empirical orthogonal function (EOF) analysis has been widely used in meteorological and climatological studies to statistically identify dominant anomaly patterns. The most dominant pattern of wintertime northern hemisphere (NH) sea-level pressure (SLP) variability, as identified in the first leading EOF, is known as "Arctic Oscillation (AO)" (see Thompson and Wallace, 1998; hereafter cited as TW98). They claimed its dynamical meaningfulness, which was then developed into a dynamical concept of "annular mode" by Thompson and Wallace (2000) and Wallace (2000). However, it is under debate whether the AO indeed represents physically meaningful variability (Wallace and Thompson 2002) or it is merely a statistical artifact. Particularly, it has been questioned whether the inphase relationship between the midlatitude anomalies over the Atlantic and Pacific is indeed a manifestation of the annularity of the AO pattern (Deser 2000; Ambaum et al. 2001; Itoh 2002a, b). Quadrelli and Wallace (2004) attempted to project several known patterns of variability onto a particular phase space defined by the first eigenvector (EOF1) and the second one (EOF2) obtained by an EOF analysis. They showed that those prominent climatological phenomena are well expressed by the linear combinations of EOF 1 and EOF2.

In the present study, an attempt is made to reexamine the leading principal components (PCs) from a viewpoint of "statistical independence". Specifically, we

Corresponding author: Atsushi Mori, Department of Astronomy and Earth Sciences, Tokyo Gakugei University, 4-1-1, Nukuikita, Koganei, Tokyo, Japan. E-mail: mori@buran.u-gakugei.ac. jp. (C2006, the Meteorological Society of Japan. apply the so-called independent component analysis (ICA) to examine whether the AO signal, which is defined as the first PC (PC1), can almost stand alone as a statistically independent signal against other PCs, or it can be decomposed into two or more statistically independent signals. ICA is a rather new statistical technique. It started to be used in the 1990s in the field of multi-dimensional signal processing (see e.g., Jutten and Herault 1988). The state of the art is given in a review by Hyvärinen and Oja (2000) and in a textbook of Hyvärinen et al. (2001). ICA has been successful in resolving observed mixed signals, including brain imaging data and voice signals. Although its applicability to climatological data has been discussed by Aires et al. (2002) in conjunction with a rotated EOF analysis, ICA is not yet familiar to our community. Accordingly, a brief introduction of the technique is given prior to its practical applications.

\section{Independent component analysis}

\subsection{ICA model}

Suppose we are attempting to retrieve $n$ original independent source signals $s_{1}(t), \cdots, s_{n}(t)$ from $m$ observed time series data $x_{1}(t), \cdots, x_{m}(t)$. Hereafter, we drop the time index $t$ for conciseness. It is usually assumed that $x_{i}$ is a linear combination of $s_{j}$, i.e.,

$$
x_{i}=a_{i 1} s_{1}+a_{i 2} s_{2}+\cdots+a_{i n} s_{n}
$$

where $a_{i j}$ are called mixing coefficients. The mixing model (1) may be written in a vector-matrix form as

$$
\mathbf{x}=\mathbf{A s},
$$

where $\mathbf{x}=\left(x_{1}, \cdots, x_{m}\right)^{T}, \mathbf{s}=\left(s_{1}, \cdots, s_{n}\right)^{T}$, and $\mathbf{A}=\left(a_{i j}\right)$. The superscript $T$ denotes the transpose of a matrix or a vector. The coefficient matrix $\mathbf{A}$ is called "mixing matrix". Just like EOF analysis, the mixing matrix A and the source signal $\mathbf{s}$ are to be determined.

One of the assumptions allowed is that each of the components of $\mathbf{s}$ are statistically independent of one another. Another assumption is that the probability density function (pdf) of any independent component $s_{i}$ must be non-Gaussian, which is discussed in subsection 2.3 in detail. The statistical model represented in (2) with those assumptions is called the Independent Component Analysis model or ICA model. For simplicity, we further assume that the unknown $\mathbf{A}$ is a square matrix. Once A or the corresponding "separating matrix" W, which is defined as the inverse of A, can be known, one can obtain the independent components $s_{i}$ by

$$
\mathbf{s}=\mathbf{W} \mathbf{x}
$$

Prior to an actual application of the ICA, it is convenient to pre-process the observed data through 
"centering" and "whitening". The former is to center the observed data $\mathbf{x}$ by subtracting its mean $\mathbf{m}=E\{\mathbf{x}\}$, where $E\{\}$ signifies expectation. The latter, applied after the centering, is to transform the vector $\mathbf{x}$ linearly, yielding a new vector $\tilde{\mathbf{x}}$ that is "white" or "sphered". Thus, all the components of $\tilde{\mathbf{x}}$ are mutually uncorrelated, and their variances are all equal to unity: $E\left\{\tilde{\mathbf{x}} \tilde{\mathbf{x}}^{T}\right\}=\mathbf{I}$, where $\mathbf{I}$ is the unit matrix. With the pre-processed data, the original formulation (3) can be rewritten as $\mathbf{s}=\tilde{\mathbf{W}} \tilde{\mathbf{x}}$, where $\tilde{\mathbf{W}}$ is the separating matrix corresponding to $\tilde{\mathbf{x}}$. Since any pair of the components of $\tilde{\mathbf{x}}$ remains uncorrelated under any orthonormal transformation, the $\tilde{\mathbf{W}}$ that we want to find must be orthonormal. In the following, $\tilde{\mathbf{W}}$ and $\tilde{\mathbf{x}}$ are simply expressed as $\mathbf{W}$ and $\mathbf{x}$, respectively.

\subsection{What is statistical independence?}

Two scalar-valued random variables $y_{1}$ and $y_{2}$ are considered "statistically independent" if any information on the value of $y_{1}$ yields no information on the value of $y_{2}$, and vice versa. Technically, their independence can be defined on the basis of their probability densities. We define that $y_{1}$ and $y_{2}$ are statistically independent if and only if joint pdf $p\left(y_{1}, y_{2}\right)$ is factorizable: $p\left(y_{1}, y_{2}\right)=p_{1}\left(y_{1}\right) \cdot p_{2}\left(y_{2}\right)$, where $p_{1}\left(y_{1}\right)$ and $p_{2}\left(y_{2}\right)$ are marginal pdfs of $y_{1}$ and $y_{2}$, respectively. The definition can be used to derive one of the most fundamental properties of independent variables. Given two functions, $h_{1}$ and $h_{2}$, we always have

$$
E\left\{h_{1}\left(y_{1}\right) \cdot h_{2}\left(y_{2}\right)\right\}=E\left\{h_{1}\left(y_{1}\right)\right\} \cdot E\left\{h_{2}\left(y_{2}\right)\right\},
$$

if $y_{1}$ and $y_{2}$ are mutually independent. In the ICA model (2), it is impossible to determine the independent source signals that satisfy (4) for any pair of arbitrarily chosen functions $h_{1}$ and $h_{2}$. In practice, it is hence important to specify a particular pair of functions according to a plausible principle as will be explained in subsection 2.3.

Taking such $h_{1}$ and $h_{2}$ to yield $h_{1}(y)=h_{2}(y)=y$, one can easily find that "uncorrelatedness" is merely a weaker form of statistical independence between random variables $y_{1}$ and $y_{2}$ :

$$
E\left\{y_{1} \cdot y_{2}\right\}-E\left\{y_{1}\right\} \cdot E\left\{y_{2}\right\}=0
$$

Note that two certain random variables are uncorrelated if they are independent, but the reverse is not necessarily true. Uncorrelatedness therefore does not necessarily mean statistical independence.

\subsection{Principle of ICA algorithm}

The Central Limit Theorem tells us that the pdf of the sum of independent random variables approaches toward a Gaussian distribution under certain condition. Given that theorem, the basic procedure of ICA is to decompose observed data into unknown independent signals $s_{i}$ by maximizing their "non-Gaussianity". The following approximation was made in evaluating nonGaussianity $J$ of a normalized random variable $y$ :

$$
J(y) \propto|E\{G(y)\}-E\{G(v)\}|,
$$

where $G$ is a non-quadratic nonlinear function of $y$, and $v$ is a specified random variable with Gaussian pdf whose variance is identical to that of $y$, i.e., unity. Hyvärinen and Oja (2000) proposed three types of functions for $G(y): G_{1}(y)=\log (\cosh (y)), G_{2}(y)=-\exp \left(-y^{2} / 2\right)$, and $G_{3}(y)=y^{4}$. We adopt $G_{1}(y)=\log (\cosh (y))$ in the following examples, although we have verified that our results still remain essentially the same if based on the other functions.

Maximizing the non-Gaussianity can be proved to lead a necessary condition for mutual independence among $s_{i}$ in the following way. A particular row of $\mathbf{W}$, say the $i$-th row $\mathbf{w}_{i}$, is assumed to be determined through maximizing the non-Gaussianity of the corresponding source signal $s_{i}\left(=\mathbf{w}_{i}^{T} \mathbf{x}\right)$ based on (5), under the constraint of $\mathbf{w}_{i}^{T} \mathbf{w}_{i}=1$. This maximization may be formulated as

$$
\frac{\partial}{\partial \mathbf{w}_{i}}\left(\left(E\left\{G\left(\mathbf{w}_{i}^{T} \mathbf{x}\right)\right\}-E\{G(v)\}\right)-\frac{\beta_{i}}{2} \mathbf{W}_{i}^{T} \mathbf{w}_{i}\right)=0,
$$

with a Lagrange multiplier $\beta_{i}$. It follows that

$$
E\left\{\mathbf{x} g\left(\mathbf{w}_{i}^{T} \mathbf{x}\right)\right\}-\beta_{i} \mathbf{w}_{i}=0,
$$

where the function $g$ is the derivative of $G$. It is noteworthy that multiplying the both sides of (7) by the transpose of another row $\mathbf{w}_{j}$ of $\mathbf{W}$ yields

$$
\begin{aligned}
& E\left\{g\left(\mathbf{w}_{i}^{T} \mathbf{x}\right) \cdot\left(\mathbf{w}_{j}^{T} \mathbf{x}\right)\right\}-\beta_{i} \mathbf{W}_{j}^{T} \mathbf{w}_{i} \\
= & E\left\{g\left(\mathbf{w}_{i}^{T} \mathbf{x}\right) \cdot\left(\mathbf{w}_{j}^{T} \mathbf{x}\right)\right\}-\beta_{i} \delta_{i j} \\
= & 0,
\end{aligned}
$$

or equivalently $E\left\{g\left(s_{i}\right) \cdot s_{j}\right\}=\beta_{i} \delta_{i j}$, because $\mathbf{W}$ is orthonormal. This corresponds to (4), if we select $h_{1}(y)=g(y)$ and $h_{2}(y)=y$. Hence, maximizing the non-Gaussianity of the $s_{i}$ leads to a necessary condition for the mutual independence among the independent components s. Since ICA model (2) is a linear model, a certain choice of $g(y)$ leads to the unique solution of $\mathbf{W}$ except an ambiguity in choosing the axes of the orthonormal transformation.

For the efficient determination of $\mathbf{W}$ based on (7), Hyvärinen (1999) developed the Fast ICA algorithm. In this algorithm, based on Newton's method, $\mathbf{w}_{i}$ can be iteratively obtained. Since the approximation used in this algorithm, however, is not always appropriate, the validity of the results should be double checked. Thus, we also evaluated $J$ with a fine grid of rotation angles of orthonormal transformation and maximized the sum of $J$ for all the components. In this manner, we have verified that the two results are identical within the accuracy of $10^{-3}$ radian except an ambiguity in the choice of axes.

\section{Three point models of AO}

Ambaum et al. (2001) and Itoh (2002a) critically argued the reality of the AO pattern, by contrasting two hypothetical models of the AO whose eigenvectors are identical. Those models are simple enough to illustrate how the ICA works. They proposed:

- AO Model-1

$$
\left\{\begin{array}{l}
x_{n}=2 s_{1} \\
x_{p}=-s_{1}+s_{2} \\
x_{a}=-s_{1}-s_{2},
\end{array}\right.
$$

- AO Model-2

$$
\left\{\begin{array}{l}
x_{n}=s_{3}+s_{4} \\
x_{p}=-s_{3}-s_{4} .
\end{array}\right.
$$

In these models, $x_{n}, x_{p}$, and $x_{a}$ signify surface pressure anomalies at the centers of action of the AO pattern located in the Arctic, North Pacific, and North Atlantic, respectively. We assume that $s_{1}, s_{2}, s_{3}$, and $s_{4}$ are mutually independent in a statistical sense and they represent individual SLP anomaly signals with certain physical entity.

Since the first eigenvector (EOF 1$)$ is proportional to $(2,-1,-1)$ for each of the models, a conventional EOF analysis fails to distinguish intrinsic structure between the two models. However, Itoh (2002b) showed that those two models are distinguishable if the PCs or the scores of EOF1 and EOF2 are plotted on a phase space as shown in Fig. 1. Following his paper, each of $s_{1}, s_{2}, s_{3}$, and $s_{4}$ is given with 1,000 independent random numbers distributed uniformly between -1 and 1 . It is apparent in Fig. 1 that the scores of EOF 1 and EOF2 are statistically independent for the AO Model-1, but not for the AO Model-2. The joint pdf for the latter model cannot be expressed as the product of the pdfs of the two EOFs. Meanwhile, as shown in Fig. 2, the ICA successfully extracts the two statistically independent source signals for each of the models as the first and second inde- 
a)

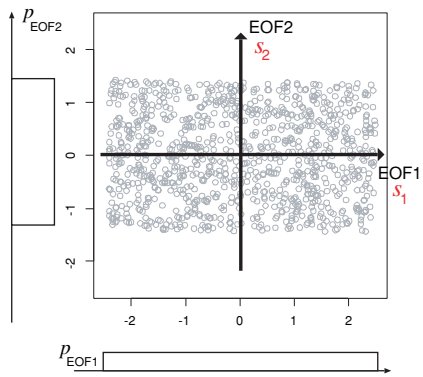

b)

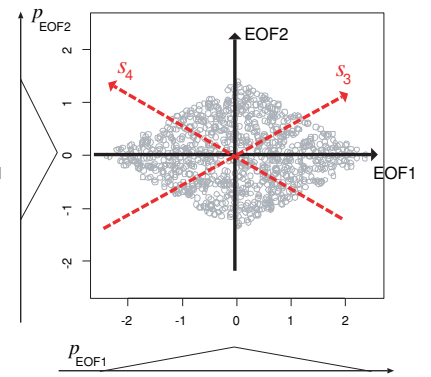

Fig. 1. Scores on EOF1-EOF2 plain for (a) the AO Model1 and (b) the AO Model-2. Pdfs for the first and the second PCs are schematically plotted at the bottom and on the left, respectively, of each of the panels.

a)

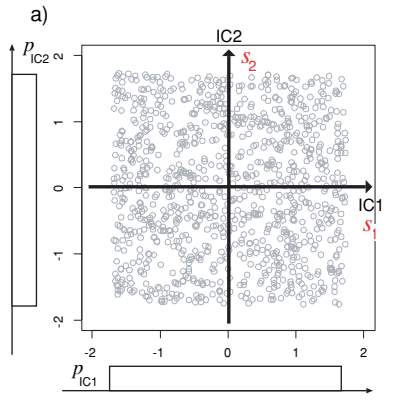

b)

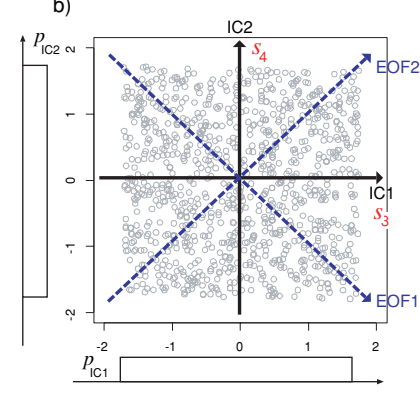

Fig. 2. Estimated source signals are plotted on the first and the second independent components (IC1 and IC2) phase plane for (a) the AO Model-1 and (b) the AO Model-2. The marginal pdfs of the IC1 and IC2 are schematically plotted at the bottom and on the left of each of the panels.

pendent components (ICs). Note that the pdfs of the PCs for the AO Model-2 are more Gaussian those that of ICs. The pdf of any linear combination of $s_{3}$ and $s_{4}$ should be more Gaussian than that of $s_{3}$ or $s_{4}$ itself.

\section{Application to observed data}

Prior to our practical application of ICA to observed SLP anomaly data $\mathbf{x}_{o b s}$, it is computationally efficient to reduce the degrees of freedom. For this purpose, an EOF analysis was performed to $\mathbf{x}_{o b s}$ firstly, and the $N$ leading PCs and the corresponding EOFs were retained to approximate the data as $\mathbf{x}_{o b s} \sim \mathbf{x}_{a p} \equiv \mathbf{V} \mathbf{p}$, where the vector $\mathbf{p}$ and the matrix $\mathbf{V}$ contain those $N$ PC time series and the associated EOFs, respectively. This EOF-based approximation is also effective in extracting phenomena with a large amplitude and in reducing noise in the data to which the Fast ICA algorithm is susceptible. Thus, only the limited number $(N)$ of the leading PCs was incorporated into ICA: $\mathbf{p}=$ As where $\mathbf{s}$ denotes the corresponding source signals of the $N$ independent components. Combining the above equations, one can readily obtain $\mathbf{x}_{a p}=\mathbf{B s}$ with $\mathbf{B}=\mathbf{V A}$. A column of the matrix $\mathbf{B}$, called a basis vector, represents an anomaly pattern associated with a particular IC. Once $\mathbf{A}$ and $\mathbf{B}$ are known, one can express any of the selected EOFs as a linear combination of the basis vectors based on $\mathbf{V}=\mathbf{B A}^{-1}$. If the PCs, including the AO signal, are superposition of other statistically independent signals, the pdfs of the PCs would be closer to Gaussian than the source signals, and ICA would be applicable.

In an ICA application, we used atmospheric reanalysis data for the period 1950-2002 provided by the U.S.

a) Basis Vector for IC1
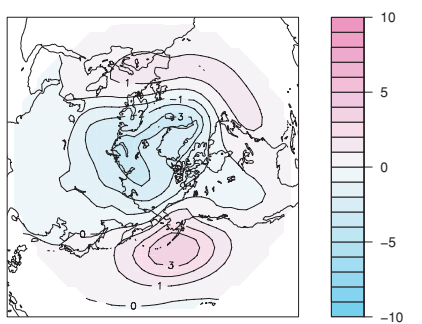

b) Basis Vector for IC2

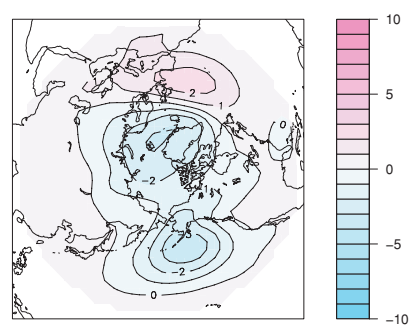

Fig. 3. Basis vectors for (a) IC1 and (b) IC2, as obtained by ICA for NH monthly SLP for 1950-2002. The presented anomalies (hPa) are for standard deviations of ICs.
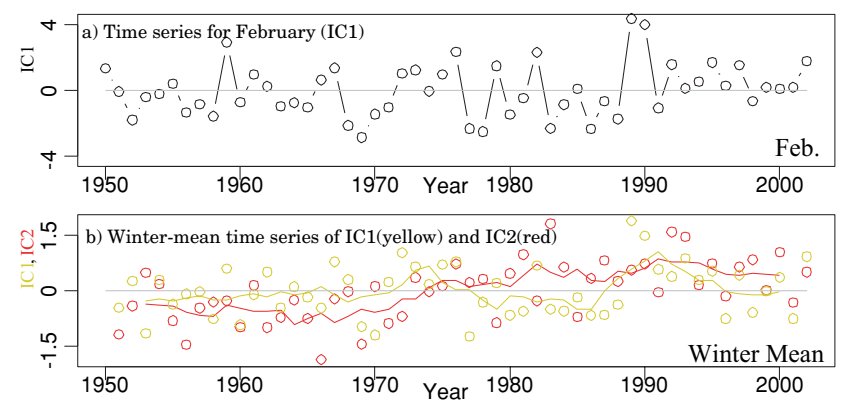

Fig. 4. (a) IC1 time series for February. (b) Winter-mean time series (circles) and the corresponding 5-year running mean (solid line) of $\mathrm{IC} 1$ (yellow) and IC2(red). Winter is defined as November through April. The IC1 and IC2 time series have been normalized annually so that their standard deviations become unity when all the 12 calendar months are incorporated.

National Centers of Environmental Prediction (NCEP) and the National Center for Atmospheric Research (NCAR). Following TW98, we first performed a conventional EOF analysis on the monthly anomaly fields of SLP poleward of $20^{\circ} \mathrm{N}$. Although many of the prominent phenomena can be expressed by the linear combination of PC1 and the second PC (PC2) (Quadrelli and Wallace 2004), we choose the degree of freedom as $N=3$ in order to check the robustness of the results. The data for all calendar months were incorporated as in Thompson and Wallace (2000). Results for winter, in which shows a larger variability of SLP, are basically the same. As discussed in subsection 2.3, however, the signals should not be Gaussian random variables. The pdfs of the ICs obtained for winter are much closer to Gaussian distribution, while we have verified the ICs obtained for all calendar months are not Gaussian variables with significance level of more than 99\% (not shown).

Figures 3 and 4 summarize our ICA results based on the three leading PCs. To a good approximation, the AO time series (i.e., PC1) can be represented as a superposition of two ICs (IC1 and IC2). IC1 shown in Fig. 3a accounts for $67 \%$ of the variance of PC1. The corresponding basis vector represents an anomaly pattern similar to the interannual seesaw between the intensities of the surface Aleutian and Icelandic lows (AIS; see Honda et al. 2001). The dominance of IC1 in PC1 is consistent with Honda and Nakamura (2001), who showed the AO signature in late winter is basically the AIS. In fact, the IC1 time series, which is normalized annually, attains the largest variance (2.60) in February, the peak time of the AIS. Furthermore, the IC1 time series for February shown in Fig. 4a is strongly correlated $(r=$ 0.90) with the AIS index defined by Nakamura and 
Honda (2002). Indeed, the linear regression map of SLP with the February IC1 time series well reproduces the AIS signature (not shown). In contrast, the basis vector for IC2 (Fig. 3b), which accounts for 31\% of the variance of $\mathrm{PC} 1$, represents an in-phase relationship between the AL and IL that resembles the "cold ocean-warm land (COWL) pattern" (Wallace et al. 1995). Consistently, the annually normalized IC2 time series attains its largest interannual variance (2.68) in January, and its winter mean time series is characterized by a marked upward trend over the last three decades, while IC1 does not show any significant trend (Fig. 4b). In fact, the correlation coefficient between the winter mean IC2 and the COWL pattern index of 1951-1995, defined by the land surface temperature (New et al. 2000) poleward of $20^{\circ} \mathrm{N}$, reaches as high as 0.69 .

Concerning the decomposition of $\mathrm{PC} 2,28 \%$ of its variance is attributed to IC1, while $68 \%$ of the variance is owing to IC2. And the third IC corresponds to the third PC almost one by one. Thus, IC1 and IC2 can almost entirely explain the large variance of SLP represented by $\mathrm{PC} 1$ and $\mathrm{PC} 2$. The results described above have been verified to be essentially the same as those for $N=2$ (not shown).

\section{Summary and discussion}

The signals generated by physically independent phenomena are expected to be statistically independent of one another, and therefore, they are also expected to be uncorrelated one another. For this reason, an EOF analysis is used to seek physically meaningful signals and spatial patterns under the supposition of uncorrelatedness among the PC time indices. As has been already pointed out, however, orthogonality of the spatial structure of the phenomena is a too strong constraint in some occasions. The present study cautions against possible misinterpretation of PCs, from a viewpoint of statistical independence.

We argue that ICA is a useful tool to check this point. As shown in the example in section 3, ICA extracts the original source signals successfully. Although we admit that ICA never guarantees the physical entity of its results, it is noteworthy that the surface AO signal can be decomposed through ICA into signals of two known patterns of variability that are statistically independent of one another.

It is not our intention to claim that a conventional EOF analysis never extracts physically meaningful patterns. For example, the AIS is well represented in the leading EOF of wintertime NH circulation anomalies in the free troposphere (Honda and Nakamura 2001; Ambaum et al. 2001). Furthermore, it is well known (e.g., Thompson and Wallace 2000) that the leading EOF of stratospheric pressure anomalies in the winter hemisphere unambiguously represents the predominant variability in the polar-vortex intensity (stratospheric "annular mode"). The particular variability can be extracted as IC1 when ICA is applied to the wintertime stratosphere (not shown).

The rotated EOF analysis and ICA are similar in the sense that they manipulate the selected PCs and EOFs to seek potentially more meaningful patterns. However, their manipulations are based on different viewpoints. The rotation of EOFs is designed for extracting regional anomaly patterns such as the North Atlantic Oscillation (NAO) or Pacific/North American (PNA) pattern, while ICA is designed for finding statistically independent signals.

\section{Acknowledgments}

This work has been motivated by the work by Prof.
Itoh of Kyushu University. The calculation was done using the statistical package "R" and "Fast ICA package". This research was partly supported by a Grant-in-Aid from the Ministry of Education, Culture, Sports, Science and Technology of Japan (No. 16654073).

\section{References}

Aires, F., W. B. Rossow, and A. Chédin, 2002: Rotation of EOFs by the independent component analysis: Toward a solution of the mixing problem in the decomposition of geophysical time series, J. Atmos. Sci., 59, 111-123.

Ambaum, M. H. P., B. J. Hoskins, and D. B. Stephenson, 2001: Arctic Oscillation or North Atlantic Oscillation?, J. Climate, 14, 3495-3507.

Deser, C., 2000: On the teleconnectivity of the "Arctic Oscillation", Geophys. Res. Lett., 27, 779-782.

Honda, M., H. Nakamura, J. Ukita, I. Kousaka, and K. Takeuchi, 2001: Interannual seesaw between the Aleutian and Icelandic Lows. Part I: seasonal dependence and life cycle, J. Climate, 14, 1029-1042.

Honda, M., and H. Nakamura, 2001: Interannual seesaw between the Aleutian and Icelandic lows. Part II: Its significance in the interannual variability over the wintertime Northern Hemisphere, J. Climate, 14, 4512-4529.

Hyvärinen, A., 1999: Fast and robust fixed-point algorithms for independent component analysis, IEEE Transactions on Neural Networks, 10(3), 626-634.

Hyvärinen, A., and E. Oja, 2000: Independent component analysis: algorithms and applications, Neural Networks, 13, 411-430.

Hyvärinen, A., J. Karhunen, and E. Oja, 2001: Independent Component Analysis, John Wiley \& Sons, pp 481.

Itoh, H., 2002a: True versus apparent Arctic Oscillation, Geophys. Res. Lett., 29(8), DOI:10.1029/2001GL013978.

Itoh, H., 2002b: Mikake no Hokkyokushindou to Shin no Hokkyokushindou (in Japanese), Gross Wetter, 40, 98111.

Jutten, C., and J. Herault, 1988: Independent components analysis (INCA) versus principal components analysis. Signal Processing IV: Theories and Applications, J. L. Lacoume, A. Chehikian, N. Martin, and J. Malbos, Eds., Elsevier Science, 643-646.

Nakamura, H., and M. Honda, 2002: Interannual seesaw between the Aleutian and Icelandic Lows Part III: Its influence upon the stratospheric variability, J. Meteor. Soc. Japan, 80, 1051-1067.

New, M., M. Hulme, and P. Jones, 2000: Representing twentiethcentury space-time climate variability. Part II: Development of 1901-96 monthly grids of terrestrial surface climate, J. Climate, 13, 2217-2238.

Quadrelli, R., and J. M. Wallace, 2004: A simplified linear framework for interpreting patterns of northern hemisphere wintertime climate variability, J. Climate, 17, 3728-3744.

Thompson, D. W. J., and J. M. Wallace, 1998: The Arctic Oscillation signature in the wintertime geopotential height and temperature fields, Geophys. Res. Lett., 25, 1297-1300.

Thompson, D. W. J., and J. M. Wallace, 2000: Annular modes in the extratropical circulation. Part I: Month-to-month variability, J. Climate, 13, 1000-1016.

Thompson, D. W. J., J. M. Wallace, and G. C. Hegerl, 2000: Annular modes in the extratropical circulation. Part II: Trends, J. Climate, 13, 1018-1036.

Wallace, J. M., 2000: North Atlantic Oscillation/Annular Mode: Two paradigms-one phenomenon, Quart. J. Roy. Meteor. Soc., 126, 791-805.

Wallace, J. M., and D. W. J. Thompson, 2002: The Pacific center of action of the Northern Hemisphere annular mode: Real or Artifact?, J. Climate, 15, 1987-1991.

Wallace, J. M., Y. Zhang, and J. A. Renwick, 1995: Dynamic contribution to hemispheric mean temperature trends, Science, 270, 780-783.

Manuscript received 5 October 2005, accepted 28 November 2005 SOLA: http://www.jstage.jst.go.jp/browse/sola/ 